Publ. RIMS, Kyoto Univ.

15 (1979), 773-785

\title{
Injective Envelopes of Operator Systems
}

\author{
By \\ Masamichi HAMANA*
}

\begin{abstract}
Albstract
We show the existence and uniqueness of a minimal injective operator system (resp. minimal unital $C^{*}$-algebra) "containing" a given operator system $\mathrm{V}$, which will be called the injective (resp. $C^{*}$ ) envelope of $V$. This result can be applied to prove the existence of the Šilov boundary in the sense of Arveson, which was left open in [1].
\end{abstract}

\section{$\S 1$. Introduction}

We will use terminologies in Arveson [1] and Choi-Effros [2], [3] without further explanation, and we will denote the set of all bounded operators on a Hilbert space $H$ by $B(H)$. For a subset $S$ of a unital $C^{*}$-algebra $A, C^{*}(S)$ stands for the $C^{*}$-subalgebra of $A$ generated by $S$ and the unit 1. If, in addition, $S$ is self-adjoint, linear, and contains 1 , $S$ can be regarded as an operator system in the obvious fashion. In fact consider a faithful $*$-representation $\{\pi, H\}$ of $A$ and identify $S$ with the operator system $\pi(S) \subset B(H)$. This identification is justified since $\left.\pi\right|_{S}$ : $S \rightarrow \pi(S)$ is a unital (= unit-preserving) complete order isomorphism, and will be made throughout the paper.

Let $V \subset B(H)$ be an operator system and let $\kappa: V \rightarrow B(K)$ be a unital complete order injection (i.e. a unital complete order isomorphism of $V$ onto $\kappa(V) \subset B(K))$. Then, although $V$ and $\kappa(V) \subset B(K)$ have the same structure as matrix order unit spaces, generally we can not guess any relation between the $C^{*}$-algebras $C^{*}(V) \subset B(H)$ and $C^{*}(\kappa(V)) \subset B(K)$ generated by them. So it will be an interesting problem to find a minimal $C^{*}$-algebra (if it makes sense) which is generated by the operator system which is unitally completely order isomorphic to the given operator system $V$.

Communicated by H. Araki, September 28, 1977.

* Department of Mathematics, Faculty of Education, Toyama University. 
We will show in the following that such a $C^{*}$-algebra exists uniquely and that it is $*$-isomorphic to the quotient $C^{*}$-algebra $C^{*}(V) / J$, where $J$ is the Šilov boundary for $V$ in the sense of Arveson [1, Definition 2.1.3]. We call the $C^{*}$-algebra the $C^{*}$-envelope of $V$. Thus an operator system determines its $C^{*}$-envelope uniquely. Conversely, it may be said that any unital $C^{*}$-algebra $A$ is determined by its self-adjoint linear subspace $V$, containing 1 , which has $A$ as its $C^{*}$-envelope (or equivalently, which has $\{0\}$ as its Silov boundary): If $\kappa$ is a unital complete order isomorphism of $V$ onto an operator system $V_{1} \subset B\left(H_{1}\right), \kappa$ extends uniquely to a $*$-isomorphism $\hat{\kappa}$ of $A$ onto $C^{*}\left(V_{1}\right) / J_{1}$ so that $\hat{\kappa}=\pi \circ \kappa$, where $J_{1}$ is the Šilov boundary for $V_{1}$ and $\pi: V_{1} \hookrightarrow C^{*}\left(V_{1}\right) \rightarrow C^{*}\left(V_{1}\right) / J_{1}$ is the canonical map. This fact, which is no other than the uniqueness of the $C^{*}$ envelope of $V$, was proved by Arveson under an additional hypothesis [1, Theorem 2. 2.5]. (There he does not assume that $V$ is self-adjoint; but without loss of generality, we may assume so.)

To solve the above problem we introduce the injective envelope of an operator system, which generalizes the injective envelope defined for a unital $C^{*}$-algebra [4].

The author is grateful to the referee for his valuable suggestions.

\section{$\S$ 2. Definitions and Preliminalies}

Throughout this section $V \subset B(H)$ will denote a fixed operator system.

Definition 2. 1. An extension of $V$ is a pair $(W, \kappa)$ of an operator system $W$ and a unital complete order injection $\kappa: V \rightarrow W$.

Definition 2.2. $(W, \kappa)$ is an injective (resp. $C^{*}$-) extension of $V$ iff $W$ is an injective operator system (resp. unital $C^{*}$-algebra such that $\left.C^{*}(\kappa(V))=W\right)$.

Definition 2.3. $(W, \kappa)$ is an essential extension of $V$ iff, given any operator system $Z$ and any unital completely positive map $\varphi: W \rightarrow Z$, $\varphi$ is a complete order injection whenever $\varphi \circ \kappa$ is. 
Remark. This definition of essential extensions of operator systems is consistent with that of unital $C^{*}$-algebras in [4]. In fact, let $A$ and $B$ be unital $C^{*}$-algebras and $\kappa: A \rightarrow B$ a unital complete order injection. Then, if the extension $(B, \kappa)$ is essential, $\kappa$ is a $*$-monomorphism (=oneto-one $*$-homomorphism). (See the proof of Theorem 4.1.)

Definition 2. 4. $(W, \kappa)$ is a rigid extension of $V$ iff, given any completely positive map $\varphi: W \rightarrow W, \varphi \circ \kappa=\kappa$ implies $\varphi=\mathrm{id}_{W}$ (the identity map on $W$ ).

Remark. The essentiality of $(W, \kappa)$ implies the rigidity of $(W, \kappa)$, and further provided that $(W, \kappa)$ is injective, they are equivalent. (See Lemma 3.7 and the remark succeeding to Lemma 3.7.)

Definition 2. 5. $(W, \kappa)$ is an injective (resp. $C^{*}$-) envelope of $V$ iff it is both injective and essential (resp. $C^{*}$ - and essential) extension of V.

Definition 2. 6. Let $V$ and $V_{1}$ be operator systems such that there exists a unital complete order isomorphism $\iota: V \rightarrow V_{1}$. Given extensions $(I V, \kappa)$ and $\left(W_{1}, \kappa_{1}\right)$ of $V$ and $V_{1}$, respectively, $(W, \kappa) \sim\left(W_{1}, \kappa_{1}\right)$ iff there exists a unital complete order isomorphism $\hat{\iota}: W \rightarrow W_{1}$ such that $\hat{\imath} \circ \kappa=\kappa_{1} \circ \ell$.

The injective envelope (resp. $C^{*}$-envelope) of $V$ can be regarded as a minimal object in the family of all injective extensions of $V$ or a maximal one in the family of all essential extensions of $V$ (resp. a minimal one in the family of all $C^{*}$-extensions of $V$ ). (Cf. Lemma 3.6, Theorem 4.1, Corollary 4.2 below.)

We list a few known results which will be used later. A unital complete order isomorphism between unital $C^{*}$-algebras is an algebraic *-isomorphism [3], so that an operator system is unitally completely order isomorphic to at most one unital $C^{*}$-algebra. Any injective operator system $W$ is unitally completely order isomorphic to a unique injective monotone complete $C^{*}$-algebra $B[3$, Theorem 3.1]. Hence $W$ and $B$ are identified as matrix order unit spaces. Henceforth this identification will be made without referring; thus a unital complete order isomorphism between 
injective operator systems will be regarded as a *-isomorphism between $C^{*}$-algebras,

\section{$\S$ 3. Minimal Projections on an Injective Operator System}

Let $V \subset W \subset B(H)$ be any operator systems with $W$ injective.

Definition 3. 1. A linear map $\varphi: W \rightarrow W$ is a V-projection on $W$ iff it is unital, completely positive, idempotent $\left(\varphi^{2}=\varphi\right)$ and $\left.\varphi\right|_{V}=\mathrm{id}$.

Definition 3.2. A seminorm $p$ on $W$ is a $V$-seminorm on $W$ iff $p=\|\psi(\cdot)\|$ for some completely positive map $\psi: W \rightarrow W$ with $\left.\psi\right|_{V}=\mathrm{id}_{v}$.

Definition 3. 3. Given $V$-projections $\varphi, \psi$ on $W$ (resp. $V$-seminorms $p, q$ on $W$ ), define a partial ordering $<$ (resp. $\leqq$ ) by $\varphi<\psi$ (resp. $p \leqq q)$ iff $\varphi \circ \psi=\psi \circ \varphi=\varphi$ (resp. $p(x) \leqq q(x)$ for all $x$ in $W$ ).

A $V$-projection (resp. $V$-seminorm) which is minimal with respect to this partial ordering $<$ (resp. $\leqq$ ) will be called a minimal $V$-projection (resp. minimal $V$-seminorm).

Lemma 3.4. Any decreasing net $\left\{p_{i}\right\}$ of $V$-seminorms on $W$ has a lower bound.

Proof. We note that the unit ball of $B(W, B(H))$, the Banach space of all bounded linear maps of $W$ into $B(H)$, is compact in the point- $\sigma$-weak topology.

Let $\varphi_{i}: W \rightarrow W$ be completely positive maps such that $p_{i}=\left\|\varphi_{i}(\cdot)\right\|$ and $\left.\varphi_{i}\right|_{V}=\mathrm{id}_{V}$. The injectivity of $W$ implies the existence of a completely positive idempotent linear map $\psi$ of $B(H)$ onto $W$. Regarding $\left\{\varphi_{i}\right\}$ as a subset of the unit ball of $B(W, B(H))$, the above remark shows that there are a subnet $\left\{\varphi_{j}\right\}$ of $\left\{\varphi_{i}\right\}$ and a $\varphi_{0} \in B(W, B(H))$ such that $\varphi_{j}(x) \rightarrow$ $\varphi_{0}(x) \sigma$-weakly for all $x$ in $W$. Then it is immediately seen that $\varphi_{0}$ is completely positive and $\left.\varphi_{0}\right|_{V}=\mathrm{id}_{V}$, so that the $\operatorname{seminorm} p: x \mapsto\left\|\psi \circ \varphi_{0}(x)\right\|$ is a $V$-seminorm on $W$. Moreover we have for all $x$ in $W$,

$$
p(x)=\left\|\psi \circ \varphi_{0}(x)\right\| \leqq\left\|\varphi_{0}(x)\right\| \leqq \lim \sup \left\|\varphi_{j}(x)\right\|=\lim p_{i}(x) \text {. Q.E.D. }
$$


This lemma and Zorn's lemma imply the existence of a minimal $V$ seminorm $p_{0}$ on $W$.

Theorem 3.5. Let $V \subset W \subset B(H)$ be as above. Then there exists a minimal $V$-projection on $W^{\top}$.

Proof. Let $\varphi: W \rightarrow W$ be a completely positive map such that $p_{0}(x)$ $=\|\varphi(x)\|, x \in W$, and let $\varphi^{(n)}=\left(\varphi+\varphi^{2}+\cdots+\varphi^{n}\right) / n, n=1,2, \cdots$. Then it follows from a reasoning similar to that in Lemma 3.4 that there exist a subnet $\left\{\varphi^{\left(n_{i}\right)}\right\}$ of $\left\{\varphi^{(n)}\right\}$ and a completely positive map $\varphi_{0} \in B(W, B(H))$ such that $\varphi^{\left(n_{i}\right)}(x) \rightarrow \varphi_{0}(x) \quad \sigma$-weakly for all $x$ in $W$. As in Lemma 3.4 take a completely positive idempotent linear map $\psi$ of $B(H)$ onto $W$. Then

$$
\begin{aligned}
\left\|\psi \circ \varphi_{0}(x)\right\| & \leqq\left\|\varphi_{0}(x)\right\| \leqq \lim \sup \left\|\varphi^{\left(n_{i}\right)}(x)\right\| \\
& \leqq\|\varphi(x)\|=p_{0}(x), \quad x \in W,
\end{aligned}
$$

so the minimality of $p_{0}$ implies that $\left\|\psi \circ \varphi_{0}(x)\right\|=p_{0}(x)$, hence that $\lim \sup \left\|\varphi^{\left(n_{i}\right)}(x)\right\|=\|\varphi(x)\|$. Therefore

$$
\left\|\varphi(x)-\varphi^{2}(x)\right\|=\lim \sup \left\|\varphi^{\left(n_{i}\right)}(x-\varphi(x))\right\|=0, \quad x \in W,
$$

so that $\varphi$ is a $V$-projection on $W$.

The proof of the minimality of $\varphi$ is exactly the same as that of the case where $V$ is a unital $C^{*}$-algebra [4, Theorem 3.4]. Q.E.D.

Lemma 3. 6. Let $V \subset W \subset B(H)$ be as above and let $\varphi$ be a minimal $V$-projection on $W$. Then the extension $(\operatorname{Im} \varphi, j)$ of $V$, where $j: V \rightarrow \operatorname{Im} \varphi=\varphi(W)$ is the inclusion map, is rigid.

Proof. Let $\psi: \operatorname{Im} \varphi \rightarrow \operatorname{Im} \varphi$ be any completely positive map such that $\psi \circ j=j$. Putting $(\psi \circ \varphi)^{(n)}=\left(\psi \circ \varphi+\cdots+(\psi \circ \varphi)^{n}\right) / n$, an argument similar to above implies the existence of a subnet $\left\{(\psi \circ \varphi)^{\left(n_{j}\right)}\right\}$ of $\left\{(\psi \circ \varphi)^{(n)}\right\}$ such that $\lim \sup \left\|(\psi \circ \varphi)^{\left(n_{j}\right)}(x)\right\|=\|\varphi(x)\|, x \in W$. Hence we have for each $x \in \operatorname{Im} \varphi$,

$$
\begin{aligned}
\|x-\psi(x)\| & =\|\varphi(x-(\psi \circ \varphi)(x))\| \\
& =\lim \sup \left\|(\psi \circ \varphi)^{\left(n_{j}\right)}(x-(\psi \circ \varphi)(x))\right\|=0,
\end{aligned}
$$


so that $\psi=\mathrm{id}_{\operatorname{Im} \varphi}$.

Q.E.D.

Lemma 3. 7. Let $(Z, \lambda)$ be an injective extension of an operator system $V$. Then $(Z, \lambda)$ is rigid iff it is essential.

Proof. Necessity: Let $Y$ be any operator system and $\varphi: Z \rightarrow Y$ any unital completely positive map such that $\varphi \circ \lambda$ is a complete order injection. Then we must show that $\varphi$ also is a complete order injection. Since $\lambda \circ(\varphi \circ \lambda)^{-1}: \quad \varphi \circ \lambda(V) \rightarrow Z$ is completely positive and $Z$ is injective, there is a completely positive map $\psi: Y \rightarrow Z$ such that $\psi_{(\varphi \circ \lambda)(\eta)}^{\mid}=\lambda_{0}(\varphi \circ \lambda)^{-1}$.

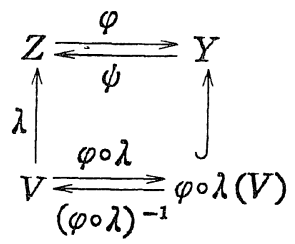

Then $\omega=\psi \circ \varphi: Z \rightarrow Z$ is a completely positive map such that $\omega \circ \lambda=\lambda$. Hence by the rigidity of $(Z, \lambda), \omega=\mathrm{id}_{Z}$, so that $\varphi$ is a complete order injection.

Sufficiency: Let $\varphi: Z \rightarrow Z$ be a completely positive map such that $\varphi \circ \lambda$ $=\lambda$. Put $\psi=\left(\operatorname{id}_{Z}+\varphi\right) / 2$. Since $\psi \circ \lambda=\lambda$, the essentiality of $(Z, \lambda)$ implies that $\psi$ is a complete order injection. We claim that $\psi$ is onto. In fact there exists a completely positive map $\left(\psi^{-1}\right)^{\wedge}: Z \rightarrow Z$ such that $\left.\left(\psi^{-1}\right)^{\wedge}\right|_{\psi(Z)}$ $=\psi^{-1}$. Then $\psi \circ\left(\psi^{-1}\right)^{\wedge}$ is idempotent and is a complete order injection since $\psi \circ\left(\psi^{-1}\right)^{\wedge} \circ \lambda=\lambda$, so that $\psi \circ\left(\psi^{-1}\right)^{\wedge}=\mathrm{id}_{Z}$. This shows that $\psi$ is onto. Hence $\psi$ is a unital complete order isomorphism of $Z$ onto itself, so it defines a $*$-automorphism of the $C^{*}$-algebra which is unitally completely order isomorphic to $Z$. From $\psi=\left(\mathrm{id}_{z}+\varphi\right) / 2$ and the extremality of a *-automorphism it follows that $\varphi=\mathrm{id}_{z}$.

Q.E.D.

Remark. Let $(W, \kappa)$ be an essential extension of an operator system $V$. Then, taking the injective envelope $(Z, \lambda)$ of $W$, whose existence will be proved below, and applying the above lemma to the injective and essential extension $(Z, \lambda \circ \kappa)$ of $V$, it follows readily that $(W, \kappa)$ is a rigid extension of $V$. 


\section{§4. Main Results}

Theorem 4. 1. Any operator system $V \subset B(H)$ has a unique injective (resp. $C^{*}$-) envelope, where the uniqueness of the extensions is up to the equivalcnce relation $\sim$ defined in Section 2.

Proof. By Theorem 3.5 applied to $V \subset W=B(H)$, there exists a minimal $V$-projection $\varphi$ on $B(H)$. Let $I_{\varphi}=\left\{x \in B(H): \varphi\left(x^{*} x\right)=\varphi\left(x x^{*}\right)\right.$ $=0\}$ and $B(H)_{\varphi}=\operatorname{Im} \varphi+I_{\varphi}$. Then $B(H)_{\varphi}$ is a unital $C^{*}$-subalgebra of $B(H), I_{\varphi}$ is a closed two-sided ideal of $B(H)_{\varphi}$, and the canonical map $\operatorname{Im} \varphi \hookrightarrow B(H)_{\varphi} \rightarrow B(H)_{\varphi} / I_{\varphi}$ is a unital complete order isomorphism ([4, Theorem 2.1, Lemma 2. 4], [3, Theorem 3.1]). Put $B=B(H)_{\varphi} / I_{\varphi}$, and let $\kappa: V \hookrightarrow \operatorname{Im} \varphi \rightarrow B(H)_{\varphi} / I_{\varphi}=B$ be the canonical map and $A$ the $C^{*}$-subalgebra of $B$ generated by $\kappa(V)$. Then Lemmas 3.6 and 3.7 imply that the extension $(B, \kappa)$ [resp. $(A, \kappa)]$ of $V$ is the desired injective (resp. $C^{*}$-) envelope of $V$.

To see the uniqueness of the injective envelope $(B, \kappa)$ take another injective envelope $\left(B_{1}, \kappa_{1}\right)$ of $V$. The injectivity of $B$ and $B_{1}$ implies the existence of completely positive maps $\iota: B \rightarrow B_{1}$ and $\iota_{1}: B_{1} \rightarrow B$ such that $\iota \circ \kappa=\kappa_{1}$ and $\epsilon_{1} \circ \kappa_{1}=\kappa$.

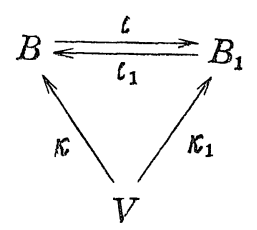

Hence $\iota_{1} \circ \iota: B \rightarrow B$ is a completely positive map with $\iota_{1} \circ 6 \circ \kappa=\kappa$, so that $\iota_{1} \circ \iota=\mathrm{jd}_{B}$ by Lemma 3.7. Similarly $\iota^{\circ} \ell_{1}=\mathrm{id}_{B_{1}}$. Hence $\iota$ is a $*$-isomorphism of $B$ onto $B_{1}$, where we regard $B_{1}$ as an injective $C^{*}$-algebra, i.e. $(B, \kappa)$ $\sim\left(B_{1}, \kappa_{1}\right)$. Note also that if $V$ is completely isometric to a $C^{*}$-algebra, then the embedding $\kappa$ (hence $\kappa_{1}$ too) becomes a $*$-monomorphism. Indeed we may then assume that $V$ is a $C^{*}$-subalgebra, containing the unit, of $B(H)$. So the map $\kappa: V \rightarrow B(H)_{\varphi} / I_{\varphi}=B$ is a $*$-monomorphism. (Since any essential extension of $V$ can be embedded in the injective envelope of $V$ by the definition, this shows that if $V$ is a $C^{*}$-algebra and $(C, \lambda)$ is an essential extension of $V$ with $C$ a $C^{*}$-algebra, then $\lambda$ becomes a *- 
monomorphism.)

The uniqueness of the $C^{*}$-envelope follows from that of the injective envelope. Indeed let $\left(A_{1}, \kappa_{1}\right)$ be another $C^{*}$-envelope of $V$ and $(D, \mu)$ the injective envelope of $A_{1}$. Then $\left(D, \mu \circ \kappa_{1}\right)$ is the injective envelope of $V$, so that from the uniqueness of the injective envelope the existence of a $*$-isomorphism $\nu$ of $B$ onto $D$ with $\nu \circ \kappa=\mu \circ \kappa_{1}$ follows.

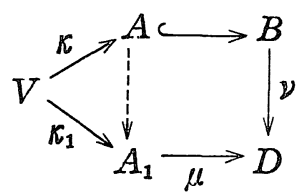

Since $\mu$ is a $*$-monomorphism as noted above,

$$
\begin{aligned}
\nu(A) & =\nu\left(C^{*}(\kappa(V))\right)=C^{*}(\nu \circ \kappa(V))=C^{*}\left(\mu \circ \kappa_{1}\left(V_{1}\right)\right) \\
& =\mu\left(C^{*}\left(\kappa_{1}\left(V_{1}\right)\right)\right)=\mu\left(A_{1}\right) .
\end{aligned}
$$

Hence we have $(A, \kappa) \sim\left(A_{1}, \kappa_{1}\right)$.

Q.E.D.

Corollary 4.2. Let $V \subset B(H)$ be an operator system and $(A, \kappa)$ the $C^{*}$-envelope of $V$. If $(B, \lambda)$ is a $C^{*}$-extension of $V$, then there is an onto *-homomorphism $\pi: B \rightarrow A$ such that $\pi \circ \lambda=\kappa$; hence $(A, \kappa)$ $\sim(B / \operatorname{Ker} \pi, q \circ \lambda)$, where $q: B \rightarrow B / \operatorname{Ker} \pi$ is the quotient homomorphism.

Proof. Without loss of generality, we may assume that $V \subset B$ $=C^{*}(V) \subset B(K)$ for some Hilbert space $K$ and $\lambda: V \rightarrow B$ is the inclusion map. Taking, as in the above proof, a minimal $V$-projection $\psi$ on $B(K)$ and letting $\rho: B(K)_{\psi} \rightarrow B(K)_{\psi} / I_{\psi}$ be the quotient map, $A_{1}=C^{*}(\rho(V))$ $\subset B(K)_{\varphi} / I_{\varphi}$ and $\kappa_{1}=\left.\rho\right|_{V}$, we obtain the $C^{*}$-envelope $\left(A_{1}, \kappa_{1}\right)$ of $V$. On the other hand, $B \subset B(K)_{\psi}$ since $B(K)_{\psi}$ is a $C^{*}$-subalgebra of $B(H)$ containing $V$ and $B=C^{*}(V)$, so that $\left.\rho\right|_{B}: B \rightarrow B(K)_{\psi} / I_{\psi}$ defines a $*$-homomorphism of $B$ onto $A_{1}$ which extends $\kappa_{1}$. Hence the uniqueness of the $C^{*}$-envelope completes the proof.

Q.E.D.

Remark. The above corollary generalizes Choi-Effros [2, Theorem 4. 1] in which $V=A$ and $\kappa$ is the identity map. 
Definition \& $\mathbb{B}^{3}$ (Arveson [1, Definition 2. 1.3]). Let $A$ be a linenr subspace of a unital $C^{*}$-algebra $B$ which contains the unit and generates $B$ as a $C^{*}$-algebra. A closed two-sided ideal $J$ of $B$ is called a boundary ideal for $A$ if the canonical quotient map $q: B \rightarrow B / J$ is completely isometric on $A$. The boundary ideal which contains every other boundary ideal is called the Šlov boundary for $A$.

We show in the following the existence of the Silov boundary, which was left open in the general situation [1]. Note first that a completely isometric linear map on $A$ extends uniquely to a completely isometric linear map on the operator system $A+A^{*}$ [1, Proposition 1.2.8] and that a unital linear map between operator systems is completely isometric iff it is a complete order injection.

'Theonem Ho Let 1 and $B$ be us above. Then there exists the Silov boundary for $A$.

Proof. By the above remark we may assume that $A=A^{*}$, i.e. $A$ is an operator system, hence that $(B, j)$ is a $C^{*}$-extension of $A$, where $j: A \rightarrow B$ is the inclusion map. Let $(C, \kappa)$ be the $C^{*}$-envelope of $A$ (Theorem 4.1). Then there is an onto $*$-homomozphism $\pi: B \rightarrow C$ such that $\pi \circ j=\kappa$ (Corollary 4.2). We verify that Ker $\pi=J$, say, is the Silov boundary for $A$. Let $\tilde{\pi}: B / J \rightarrow C$ be the *isomorphism induced by $\pi$ and $q$ : $B \rightarrow B / J$ the quotient map. Then $\tilde{\pi} \circ q \circ j=\kappa$. Hence $q \circ j=\tilde{\pi}^{-1} \circ \kappa$ is a complete order injection and $(B / J, q \circ j) \sim(C, \kappa)$. Therefore $J$ is a boundary ideal for $A$.

Let $K \subset B$ be any boundary ideal for $A$ and $q^{\prime}: B \rightarrow B / K$ the quotient nuap. Then $\left(B / K, q^{\prime} \circ j\right)$ is a $C^{*}$-extension of $A$, so again by Corollary 4. 2, there is an onto $*$-homomorphism $\rho: B / K \rightarrow B / J$ such that $\rho \circ q^{\prime} \circ j$ $=q \circ j$, i.e. $\rho(x+K)=x+J$ for all $x$ in $A$. Since $\rho$ is a $*$-homomorphism and $B$ is generated by $A$,

$$
\rho(x+K)=x+J \text { for all } i \text { in } B .
$$

In particulir, for each $x$ in $K$,

$$
0+J=\rho(0+K)=\rho(x+K)=x+J,
$$

i.e. $K \subset J$. 
Let $V,(A, \kappa),(B, \lambda)$ and $\pi$ be as in Corollary 4.2. Then, as seen in the above proof, $\operatorname{Ker} \pi$ is the Silov boundary for $\lambda(V)$. Hence the $C^{*}$-envelope of $V$ is described as a $C^{*}$-extension $(B, \lambda)$ of $V$ such that the Silov boundary for $\lambda(V)$ is $\{0\}$. Moreover the uniqueness of the $C^{*}$-envelope in Theorem 4.1 is restated as follows: Given a unital complete order isomorphism $\iota$ of an operator system $V \subset B(H)$ onto another operator system $V_{1} \subset B\left(H_{1}\right)$, there exists a unique $*$-isomorphism $\hat{\imath}$ of $C^{*}(V) / J$ onto $C^{*}\left(V_{1}\right) / J_{1}$ such that $\left.\hat{\iota} \circ q\right|_{V}=q_{1} \circ \ell$, where $J$ (resp. $J_{1}$ ) denotes the Šilov boundary $\left[\right.$ in $C^{*}(V)$ (resp. $\left.\left.C^{*}\left(V_{1}\right)\right)\right]$ for $V$ (resp. $V_{1}$ ) and $q: C^{*}(V) \rightarrow C^{*}(V) / J$ [resp. $\left.q_{1}: C^{*}\left(V_{1}\right) \rightarrow C^{*}\left(V_{1}\right) / J_{1}\right]$ means the quotient map. (Compare with [1, Theorem 2.2.5].)

We conclude this section with a remark on non-unital complete order isomorphisms. Let $V$ and $V_{1}$ be operator systems and suppose that there exists a (not necessarily unital) complete order isomorphism $\varphi: V \rightarrow V_{1}$. We want to prove that the corresponding $C^{*}$-envelopes of $V$ and $V_{1}$ are *-isomorphic (hence so are the injective envelopes of $V$ and $V_{1}$, too). We may and shall assume that $V \subset A$ (resp. $V_{1} \subset A_{1}$ ), where $A$ (resp. $A_{1}$ ) is the $C^{*}$-envelope of $V$ (resp. $V_{1}$ ). Put $\varphi(1)=b \in V_{1}$ ( 1 denotes the unit of $V$ ). Then $b$, being an order unit for $V_{1}$, is a positive invertible element of $A_{1}$. In this situation we have the following:

Proposition 4. 5. There exists a $*$-isomorphism $\alpha$ of $A$ onto $A_{1}$ which is uniquely determined by the condition: $\alpha(x)=b^{-1 / 2} \varphi(x) b^{-1 / 2}$ for all $x$ in $V$.

Proof. It is straightforward to see that $C^{*}\left(b^{-1 / 2} V_{1} b^{-1 / 2}\right)=C^{*}\left(V_{1}\right)$ $=A_{1}$ and that $\{0\}$ is the only boundary ideal for $b^{-1 / 2} V_{1} b^{-1 / 2}$. Hence $A_{1}$ is the $C^{*}$-envelope of $b^{-1 / 2} V_{1} b^{-1 / 2}$. Since $V \ni x \mapsto b^{-1 / 2} \varphi(x) b^{-1 / 2} \in b^{-1 / 2}$ $V_{1} b^{-1 / 2}$ is a unital complete order isomorphism, this map extends uniquely to a unital complete order isomorphism, hence a $*$-isomorphism of $A$ onto $A_{1}$ (Theorem 4.1).

Q.E.D.

Now we show by an example that two operator systems which are completely order isomorphic need not be unitally completely order isomorphic. Let $V$ be an operator system which is embedded in its $C^{*}$-envelope 
$A$ as a self-adjoint linear subspace containing 1 . Take an element $b \in V$ which is positive and invertible in $A$. Then $b^{-1 / 2} V b^{-1 / 2} \subset A$ may be regarded as an operator system (note that $1 \in b^{-1 / 2} V b^{-1 / 2}$ ), the map $V \ni x$ $\mapsto b^{-1 / 2} x b^{-1 / 2} \in b^{-1 / 2} V b^{-1 / 2}$ is a complete order isomorphism, and $V$ and $b^{-1 / 2}$ $V b^{-1 / 2}$ have $A$ as their $C^{*}$-envelopes (see the above proof). Hence $V$ and $b^{-1 / 2} V b^{-1 / 2}$ are unitally completely order isomorphic iff there exists a *-automorphism $\alpha$ of $A$ such that $b^{-1 / 2} V b^{-1 / 2}=\alpha(V)$.

Take as the above $V$ the linear subspace $\left\{\beta+\gamma x+\delta x^{2}: \beta, \gamma, \delta \in \mathbb{C}\right\}$ of the $C^{*}$-algebra $C([0,1])$ of all continuous functions on the unit interval $[0,1]$, where $x$ stands for the function $x \mapsto x$. Then the $C^{*}$. envelope $A$ of $V$ is $C([0,1])$, because the Šilov boundary for $V$ in the usual sense is $[0,1]$. Let $b=\beta_{0}+\gamma_{0} x+\delta_{0} x^{2} \in V$ be positive and invertible in $C([0,1])$. Since a $*$-automorphism $\alpha$ of $A$ is induced by a self-homeomorphism $h$ of $[0,1]$ so that $\alpha(f)=f \circ h, f \in A$, the equality $b^{-1 / 2} V b^{-1 / 2}$ $=\alpha(V)$ is rewritten as

$$
\begin{array}{r}
\left\{\left(\beta+\gamma x+\delta x^{2}\right) /\left(\beta_{0}+\gamma_{0} x+\delta_{0} x^{2}\right): \beta, \gamma, \delta \in \mathbb{C}\right\} \\
=\left\{\beta+\gamma / 2(x)+\delta h(x)^{2}: \beta, \gamma, \delta \in \mathbb{C}\right\} .
\end{array}
$$

But an easy computation shows that this equality does not hold provided that $\delta_{0} \neq 0$.

\section{\$5. Examples}

This section is devoted to give some simple examples of operator systems.

Example 5. 1. Let $V \subset B(H)$ be a two-dimensional operator system (i.e. $V=\mathbb{C} 1+\mathbb{C} a$ with $a^{*}=a$ and $a \notin \mathbb{C} 1$ ). Then $V$ is unitally completely order isomorphic to the commutative $C^{*}$-algebra $\mathbb{C}^{2}$. In fact, let $\lambda_{1}<\lambda_{2}$ be the end-points of the spectrum of $a$. Then the map

$$
\alpha 1+\beta a \rightarrow\left(\alpha+\beta \lambda_{1}, \alpha+\beta \lambda_{2}\right)
$$

defines a unital romplete order isomorphism of $V$ ontu $\mathbb{C}^{2}$ since it is isometric and $\mathbb{C}^{2}$ is commutative [1, Proposition 1.2.2].

Example 5.2. Let $T \in B(H)$ and let $V=\mathbb{C} 1+\mathbb{C} T+C T^{*}$. Suppose that the $C^{*}$-envelope, say $A$, of $V$ is commutative and so $A=C(X)$ with 
$X$ a compact Hausdorff space. Then $X$ is identified with the Šilov boundary (in the usual sense) for $\mathbb{C}+\boldsymbol{C} z \subset C(\operatorname{Sp} T)$, where $C(\operatorname{Sp} T)$ denotes the $C^{*}$-algebra of continuous functions on the spectrum $\operatorname{Sp} T$ of $T$ and $z$ denotes the function $\lambda \mapsto \lambda$ on $\operatorname{Sp} T$. In fact, by Theorem 4. 4, $A \cong C^{*}(V) / J$ with $J$ the Silov boundary for $V, C^{*}(V) / J$ is generated by $q(1)$ and $q(T)$, where $q: C^{*}(V) \rightarrow C^{*}(V) / J$ is the quotient homomorphism, and further $\operatorname{Sp} q(T) \subset \operatorname{Sp} T$. Hence the map $\mathbb{C} q(1)+\mathbb{C} q(T) \ni \alpha q(1)+\beta q(T)$ $\mapsto \alpha+\left.\beta z\right|_{\mathrm{Sp} q(T)} \in C(\operatorname{Sp} q(T))$ extends uniquely to a *-isomorphism of $C^{*}(V) / J$ onto $C(\operatorname{Sp} q(T))$, so that the map $V \ni \alpha 1+\beta T+\gamma T^{*} \mapsto \alpha+\beta z$ $+\gamma \bar{z} \in C(\operatorname{Sp} T)$ is a complete order injection. This shows our assertion and further that $\|\alpha 1+T\|=\sup \{|\alpha+\lambda|: \lambda \in \operatorname{Sp} T\}$ for all $\alpha \in \mathbb{C}$. The class of operators satisfying this equality was studied by Saitó [5].

Example 5. 3. Let $V \subset B(H)$ be an operator system. Then the injective envelope of $V$ is $(B(H), j)$, where $j: V \rightarrow B(H)$ is the inclusion map, iff $C^{*}(V) \supset C(H)$, the set of all compact operators on $H$, and the canonical map $V \subset C^{*}(V) \rightarrow C^{*}(V) / C(H)$ is not a complete order injection.

Now $(B(H), j)$ is the injective envelope of $V$ iff $\left(C^{*}(V), j\right)$ is the $C^{*}$-envelope of $V$ [or, what is the same, the Šilov boundary (in $C^{*}(V)$ ) for $V$ is $\{0\}]$ and $\left(B(H), j^{\prime}\right)$ is the injective envelope of $C^{*}(V)$, where $j^{\prime}: C^{*}(V) \rightarrow B(H)$ is the inclusion map (cf. the proof of Theorem 4.1). Further, noting that $C(H)$ is the smallest nonzero closed two-sided ideal of $B(H)$, we see that the Silov boundary for $V^{T}$ is $\{0\}$ iff the canonical map $V \hookrightarrow B(H) \rightarrow B(H) / C(H)$ is not a complete order injection. Thus we need only show that $\left(B(H), j^{\prime}\right)$ is the injective envelope of $C^{*}(V)$ $=A$ iff $C(H) \subset A$. If $\left(B(H), j^{\prime}\right)$ is the injective envelope of $A$, then $A^{\prime \prime}=B(H)$ by $[4$, Corollary 4.3]. Hence $C(H) \subset A$ or $C(H) \cap A$ $=\{0\}$. The latter is not the case because if $C(H) \cap A=\{0\}$, the seminorm $x \mapsto \inf _{y \in C(H)}\|x+y\|$ is an $A$-seminorm (in the sense of $[4$, Definition 3.3]) different from the norm on $B(H)$ [4, Remark 4.4]. Hence $C(H) \subset A$. Conversely, if $C(H) \subset A$, then the injective extension $\left(B(H), j^{\prime}\right)$ of $A$ is rigid, because the identity map on $B(H)$ is a unique contractive linear map of $B(H)$ into itself which fixes $C(H)$ elementwise, so that $\left(B(H), j^{\prime}\right)$ is the injective envelope of $A$ (Lemma 3.7). 


\section{References}

[1] Arveson, W., Subalgebras of $C^{*}$-algebras, Acta Math., 123 (1969), 141-224.

[2] Choi, M. -D. and Effros, E. G., The completely positive lifting problem for $C^{*}$. algebras, Ann. Math., 104 (1976), 585-609.

[3] - Injectivity and operator spaces, J. Functional Analysis, 24 (1977), 156-209.

[4] Hamana, M., Injective envelopes of $C^{*}$-algebras, J. Math. Soc. Japan, 31 (1979), 181-197.

[5] Saitô, T., Hyponormal operators and related topics, Lecture Notes in Math., 247, 533 -664, Springer-Verlag, Berlin, 1972. 
